\title{
Spatial distribution of lamin A determines nuclear stiffness and stress-mediated deformation
}

\author{
Luv Kishore Srivastava ${ }^{1}$, Zhaoping $\mathrm{Ju}^{2}$, Ajinkya Ghagre ${ }^{1}$, Allen J. Ehrlicher ${ }^{1,2 *}$
}

1. Department of Bioengineering, McGill University, Montreal H3A 0E9

2. Department of Anatomy and Cell Biology, McGill University, Montreal H3A 0C7

Abstract: The nucleus is the largest organelle and information center of the cell; while diverse cellular components have been identified as mechanotransduction elements, the deformation of the nucleus itself is emerging as a critical mechanosensory mechanism, suggesting that the nuclear stiffness is essential in determining responses to intracellular and extracellular stresses. The nuclear membrane protein, lamin $\mathrm{A}$, is known to be a dominant component in nuclear stiffening; however, the quantitative relationship between lamin A expression and nuclear deformation is still unclear. Here we measure the nuclear moduli as a function of lamin A expression and applied stress, revealing a linear dependence of bulk modulus on lamin A expression. We also find that the bulk modulus is anisotropic, with the vertical axis of the nucleus being more compliant than the minor and major axis. To examine how lamin A influences nuclear mechanics at the submicron scale we correlated the spatial distribution of lamin A with 3D nuclear envelope deformation, revealing that local areas of the nuclear envelope with higher expression levels of lamin A have correspondingly lower local deformations, and that increased lamin A expression levels result in a narrower distribution of smaller deformations. These findings describe the complex dispersion of nuclear deformations as a function of lamin A expression and distribution and implicate a role in mechanotransduction.

Introduction: The nucleus is one of the most crucial organelles and the storehouse of DNA in the cell, integrating diverse biochemical cues for proper cell function ${ }^{1}$. In addition to biochemical cues, the nucleus has an increasingly clear role as a mechanosensory structure in the cell ${ }^{2}$. Nuclear 
mechanotransduction is a process by which the nucleus detects mechanical cues and converts them to biochemical signals, which alters cell physiology. As a mechanosensor, the nucleus' stiffness determines specific deformations in response to applied stresses. The nucleus is notably stiffer than the cytoplasm $(0.5-3 \mathrm{kPa})^{3}$, with an effective Young's modulus ranging from $1-10 \mathrm{kPa}^{4,5}$; while these values appear relatively consistent in a given cell line ${ }^{6}$, multiple factors may cause nuclear stiffness to change significantly. For example, the stiffness of nuclei varies 5-fold during cell division ${ }^{7}$, in stem cells the stiffness can increase 6 -fold over the differentiation process ${ }^{8}$, and in many types of cancers the structure of the nucleus is altered and the stiffness is vastly reduced ${ }^{9}$. Many of these morphological and mechanical changes are the result of the changes in nuclear envelope architecture ${ }^{10}$.

The nuclear envelope is composed of double-membraned bilipid layers with a 30-50nm perinuclear space between the membranes. The outer nuclear membrane is connected to the endoplasmic reticulum, whereas the inner membrane is held in place by a consortium of more than 50 membrane proteins. Some of the key proteins present in the inner nuclear membrane include emerin, SUN1, SUN2, lamins, and lamin associated proteins ${ }^{11}$. Lamins are type V intermediate filaments localized under the nuclear membrane. The inner membrane is held in place by the

43 a profound effect on the stiffness of the nucleus, but lamin B shows very little or no effect on the nuclear stiffness, thus making lamin A a dominant component in nuclear mechanics ${ }^{12}$.

Numerous studies have illustrated the significance of lamin A in determining the stiffness of the nucleus. Suppression or knockdown of lamin A increases nuclear compliance, and nuclei in lamin

47 A knockout cells display $30 \%$ to $50 \%$ more deformation than WT when probed with substrate stretch or magnetic microrheology, respectively ${ }^{13}$. Previous studies have also shown that nuclei in 
49 lamin A knockout cells are more fragile, with a greater susceptibility to rupture under increased 50 intranuclear pressure ${ }^{14}$. Nuclei isolated from cells transfected with shRNA for lamin A showed

51 1.5-1.7 times more bead displacement compared to wild type nuclei in magnetic bead 52 microrheology, indicating the role of lamin A in determining the stiffness of the nucleus ${ }^{15}$.

53 Conversely, over-expression of lamin A stiffens nuclei: when 3 T3 fibroblasts were transfected

54 with lamin A and seeded on vertical nanopillars, showed 40-50\% less nanopillar induced

55 deformation in both intact and isolated nuclei ${ }^{14}$. Lamin A expression and nuclear stiffness also

56 directly affect the behavior of the cell; as an example, lamin A overexpression hinders 3D cell

57 migration through micropores ${ }^{16}$; however, it seems to facilitate $2 \mathrm{D}$ migration as wild type cells are

58 marginally faster than lamin A knockout cells ${ }^{17}$. While lamin A expression clearly influences

59 nuclear mechanics and cell behavior, the vast majority of these studies have looked at qualitative

60 treatments of lamin A, i.e., parsing cells into groups of overexpression, wild type, or knock-down.

61 However, the quantitative scaling of nuclear stiffness and corresponding nuclear deformation as a

62 function of lamin A expression remains unknown.

63 The expression of lamin A is highly variable, and changes in different stages of cell cycle ${ }^{18}$, in

64 stem cell differentation ${ }^{19}$, and as a function of microenvironment stiffness ${ }^{20}$. In addition to overall

65 expression levels, the distribution of lamin A appears heterogeneous with the formation of foci

66 and honeycomb patterns observed in the nuclear envelope ${ }^{21}$. It remains unknown how the spatial

67 heterogeneity of lamin A affects nuclear deformation. Studies to date have focused on comparing

68 gross nuclear stiffness changes in ensemble population lamin A expression levels via over-

69 expression or suppression ${ }^{22}$, but have not examined the quantitative dependence of nuclear

70 stiffness on lamin A expression, or the impact of the spatial distribution of lamin A of deformation. 
71 To understand the quantitative impact of lamin A structure on nuclear mechanics, here we measure

72 the deformability and bulk moduli of nuclei as a function of lamin A expression. We identify a

73 mechanical anisotropy of bulk compression, leading to increasing relative compliance in the major,

74 minor, and $\mathrm{z}$ axes, respectively. At the sub-micron level, we quantify the spatial distribution of

75 lamin A and show that dense lamin A structures attenuate local deformation, providing

76 heterogeneous nuclear deformations even under uniform nuclear stresses.

77

78

\section{Methodology}

Quantification of lamin A expression using antibodies and lamin A chromobody: To quantify

the total lamin A expression in the nucleus, the NIH 3T3 fibroblast cells were stably transfected quantitative metric of lamin A expression and distribution, we compared its fluorescence with antibody staining (Atto-647N Sigma) as a gold standard (see supplementary information Fig. S1).

For this, lamin A chromobody transfected cells were seeded onto gridded coverslips, fixed, permeabilized and stained with Atto-647 lamin A antibodies and then imaged with a 63X/1.4 NA

oil immersion objective on a confocal microscope (Leica SP8). Fluorescence colocalization was

87 determined using Pearson's correlation coefficient of $0.88 \pm 0.04$ for 10 cells (see supplementary information Fig. S2).

Cell culture \& modulating nuclear lamin A expression: NIH 3 T3 fibroblast cells were cultured

91 Streptomycin antibiotic. All cells were stably transfected with GFP tagged lamin A chromobody, which labels the total lamin A present on the nuclear membrane without affecting its expression. 
94 for lamin A which was a gift from Michael Davison (Addgene plasmid \# 55068), whereas to

95 suppress lamin A expression we transfected the cells with RFP tagged inducible shRNA construct

96 for lamin A (Dharmacon).

97 Quantification of nuclear bulk moduli: The cells were synchronized in $0.1 \%$ FBS containing media for 18 hours to eliminate the contribution of cell cycle on lamin A expression and distribution. They were then attached on coverslips and exposed to media with $1 \%, 2.5 \%, 5 \%$, 7.5\% and 10\% (wt/wt) 400 Da polyethylene glycol (PEG 400, Sigma) thus, exerting 847.7kPa, $936.5 \mathrm{kPa}, 1099.2 \mathrm{kPa}, 1290.3 \mathrm{kPa}$, and $1514 \mathrm{kPa}$ osmotic stresses respectively for 25 minutes to reach an equilibrium of compression ${ }^{24}$ (see supplementary information Fig. S3), followed by acquiring confocal microscopy XYZ-stacks (63x 1.4NA, Leica SP8). The cells compress due to the osmotic pressure exerted by the hypertonic solution causing an efflux of water ${ }^{25}$, and this change in volume is measured and used to determine the bulk modulus by the relation $\mathrm{B}=-\Delta \mathrm{P} /$

$106(\Delta \mathrm{V} / \mathrm{V})$ where, $\mathrm{B}=$ bulk modulus, $\Delta \mathrm{P}=$ osmotic pressure, $\Delta \mathrm{V}=$ change in volume and $\mathrm{V}=$ original 107 volume 6.

3-D volume measurement of nuclei: XYZ stacks of GFP tagged lamin A chromobody cells were imaged using a 63X/1.4 NA oil immersion objective on a Leica SP8 confocal microscope with a z-step size of $0.3 \mu \mathrm{m}$. 3-D visualization and measurement were carried out using ImageJ software. Before 3-D measurement, the nuclear images were deconvolved using the 'Iterative deconvolve 3D' plugin in ImageJ. We measured nuclear volumes by counting the number of voxels of the thresholded nuclei and then multiplied it by the size of each voxel to get the volume of the

114 thresholded region. To measure the major, minor and z-axis deformations, we acquired XYZ 
116

117

using 'z-project' plugin in image J, followed by fitting the z-projections into an ellipse and calculating the major and minor axis for different time intervals.

Nuclear strain mapping: To measure the local strain on the nuclear membrane, we captured images of the GFP tagged lamin A chromobody nucleus pre and post-exposure to $2.5 \%$ PEG 400 $(936.5 \mathrm{kPa})$. To quantify the local deformation, a custom-made MATLAB $\operatorname{code}^{26}$ was slightly modified and used. Briefly, the code utilizes a fast Fourier transform (FFT) based cross-correlation formulation in conjunction with the iterative deformation method (IDM). FIDVC calculates the displacement between the images of GFP tagged nucleus by tracking the pixel intensities of chromobody fluorescence. We mapped these local displacements with the lamin A expressions in 3-D space to determine the relationship between lamin A spatial distribution and nuclear deformation.

\section{Results:}

Lamin A stiffens the nucleus: To measure the bulk compressibility of the nuclei, we exposed cell nuclei of varying lamin A expression with different osmotic pressures using 400 Da PEG. Since PEG is impermeable across the cell membrane, it creates a hyperosmotic environment for the cell and nucleus resulting in their compression (Fig. 1a). This compression is due to the expulsion of water as the cell and nuclei regain their original volume when returned to isotonic media ${ }^{6,25}$. Previous studies have shown that under external osmotic pressure nuclear volume changes along with the concentration of intracellular macromolecules, thus exerting compressive forces on it ${ }^{6,25}$. The pressure-volume curve for nuclei of wild type NIH 3T3 fibroblast cells at different PEG 400 concentrations exerting different osmotic pressures shows that the nuclear compression was higher in low lamin A expressing cells and vice-versa (Fig. 1b) 
This is also reflected in the bulk moduli calculated from the change in the nuclear volume where the cells expressing more lamin A are relatively stiffer. The bulk modulus for the highest lamin A expressing cell at the highest exerted osmotic pressure is 3.48Mpa (Fig. 1c). A linear relationship could be seen when we plotted bulk moduli as a function of lamin A fluorescence clearly a direct relationship between lamin A expression and nuclear rigidity (Fig. 1d). We also observed the nuclear volume as a function of time for different lamin A expression cells after PEG addition and show that below lamin A fluorescence of 16.9 the rate of nuclear compression was more uniform, whereas in nuclei with lamin A expression more than 16.9 showed a sudden fall in nuclear volume below a certain level of lamin A expression (Fig. 1e \& 1f).

a

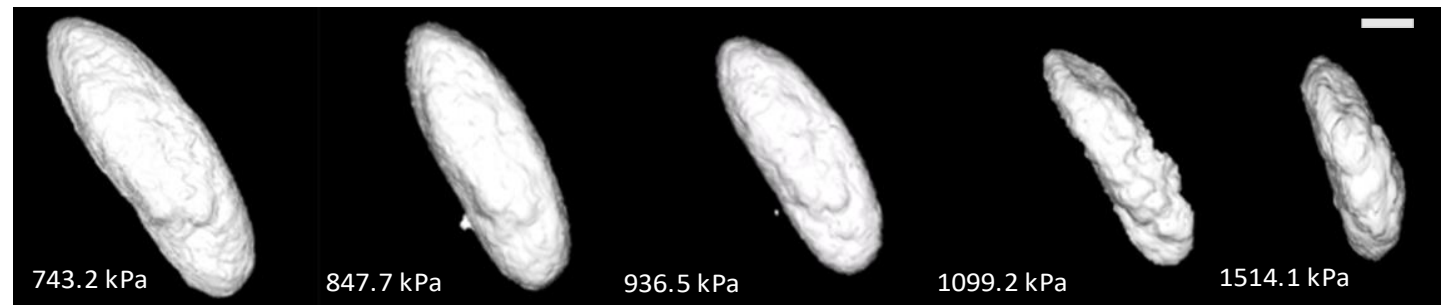

b

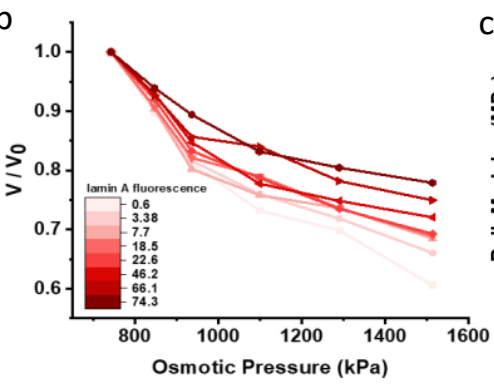

e

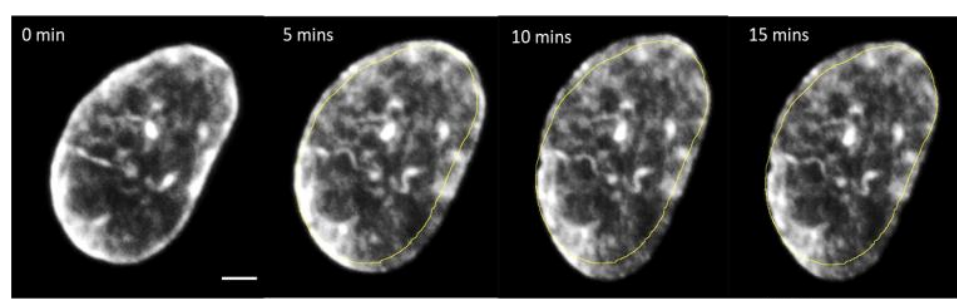

d
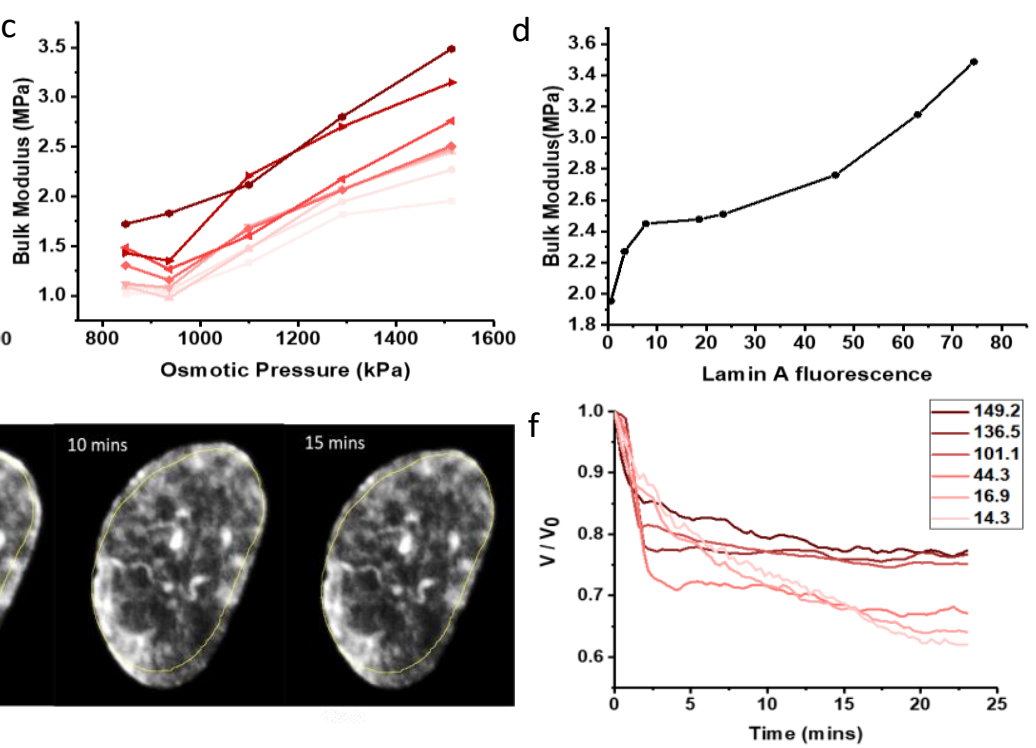

Figure 1 | Hyperosmotic compression of the nucleus in 3T3 Fibroblast cells. a, 3D reconstruction of an example nucleus within a cell at osmotic pressures of 743.2 and $1514.1 \mathrm{kPa}($ scalebar $=3 \mu \mathrm{m})$. b, Normalized nuclear volumes 
of $3 \mathrm{~T} 3$ cells transfected with lamin A chromobody for wild type cells showing that stiffness increases as the protein expression increases (fluorescence values in arbitrary units). c, Bulk moduli of 3T3 cells transfected with lamin A chromobody for wild type showing that stiffness increases as the protein expression increases $(n=8)$. d, Nuclear stiffnes in terms of bulk moduli increasing as a function of lamin A fluorescence. e, Example 3T3 fibroblast nuclei showing change in nuclear volume with time after PEG addition (scalebar $=2 \mu \mathrm{m})$. $\mathbf{f}$, Change in nuclear volume as a function of time under 1514.1kPa osmotic pressure as a function of lamin A fluorescence.

Nuclear stiffness is anisotropic. To measure the anisotropic nuclear deformation, we exposed the

a

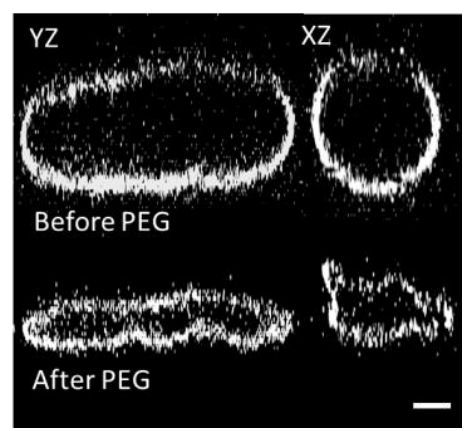

b

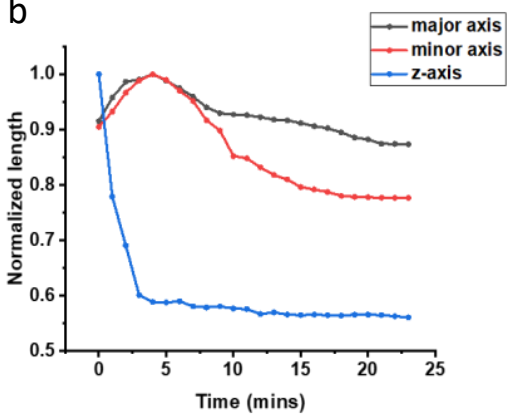

C

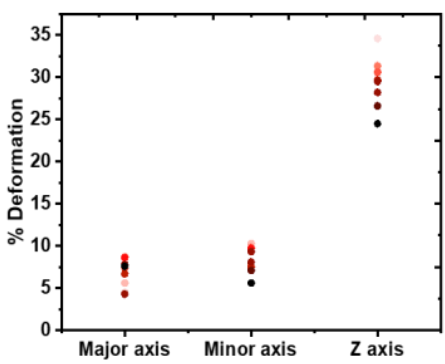

Figure 2 | Anisotropy in nuclear compression under PEG. a, Example of a wild type nuclei in Y-Z and X-Z plane before PEG addition (top) and after PEG addition (bottom) showing large deformation in the $\mathrm{z}$-axis (scalebar $=3 \mu \mathrm{m}$ ). 
171 major and minor axes expand in the beginning followed by reduction in the length showing anisotropy in nuclear

172 compression with time. c, Nuclear deformation percentage along the major, minor and z-axis in wild type nuclei for

173 different lamin A expression ( $\mathrm{n}=7$ ) showing higher deformation along $\mathrm{z}$-axis compared to the major and minor axis.

Lamin A density is spatially heterogeneous and leads to a variation in local compliance. While

177 inhomogeneity also increases with overall lamin A expression, as revealed by plotting the variance

178 of local lamin A fluorescence intensity as a function of overall expression (Fig. 3c, supplementary

179 information Fig. S5). The histogram of local lamin A fluorescence shows narrow and high peak at

180 lower lamin A fluorescence levels but the peak broadens and flattens with increase in local lamin

181 A fluorescence levels indicating that lamin A distribution gets wider or heterogenous with increase

182 in lamin A expression (Fig. 3d). We also observed the bin span from Fig. 3d as a function of total

183 lamin A expression to show that the range of lamin A fluorescence covered increased with lamin

184 A expression showing a greater heterogeneity and vice-versa (Fig. 3e).

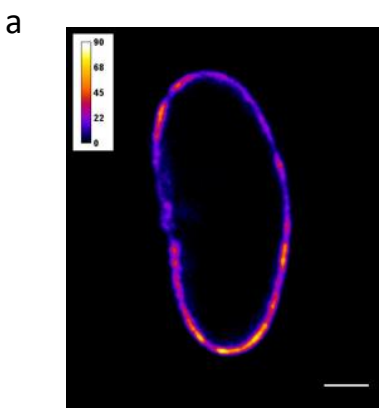

b

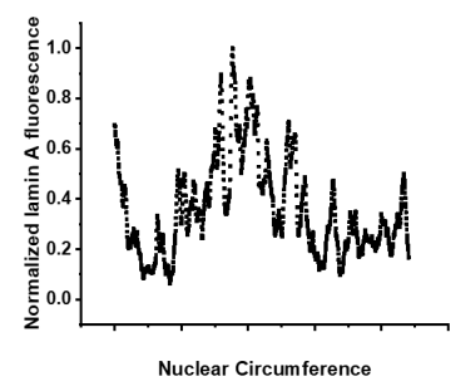

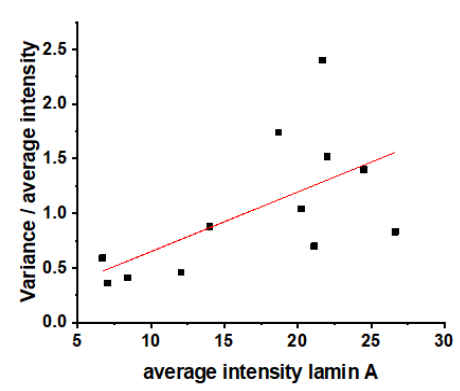

d

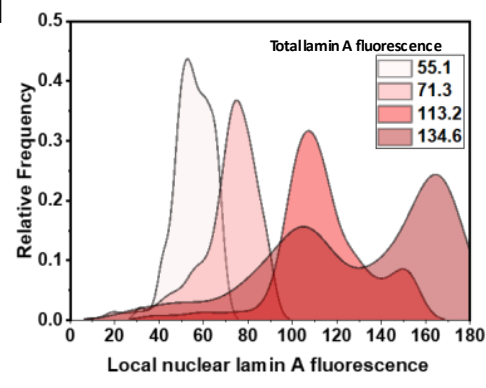

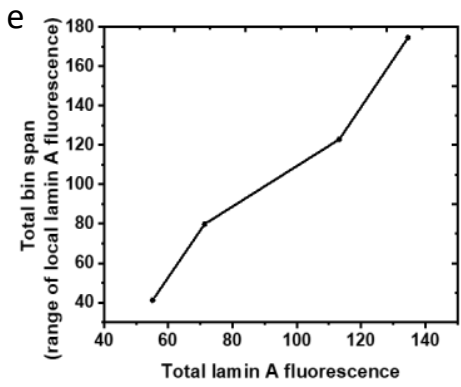


Figure 3 Lamin A is spatially heterogeneous in the nucleus. a, Example 3T3 fibroblast cells transfected with lamin A chromobody showing variability in the spatial distribution along a cross-section nuclear membrane (scalebar $=3 \mu \mathrm{m}) . \mathbf{b}$, Quantification of lamin A heterogeneity along the nuclear membrane circumference in panel $\mathbf{a}$. $\mathbf{c}$, Normalized variance of lamin A chromobody fluorescence as a function of total lamin A expression in different nuclei shows that higher expression of lamin A increases the heterogeneity of distribution $(\mathrm{n}=12)$. d, The histogram gets wider with increasing total lamin A fluorescence showing a wider range of local lamin A fluorescence or heterogeneity when overall lamin A expression increases. e. The bin span correlates linearly with lamin A expression showing a more heterogeneous and wider distribution at high lamin A fluorescence and vice-versa.

We then characterized the relationship between local lamin A expression and local nuclear deformation by quantifying the lamin A distribution via fluorescence and the nuclear deformation map in the X-Y and Z plane along the nuclear membrane using FIDVC (Fig. 4a, 4b, and 4c). These images suggest an inverse relationship between spatial variations of lamin A and deformation. To quantify the spatial variations, we plotted lamin A fluorescence with respective deformations along the nuclear circumference, showing the deformation as a function of the fraction of lamin A present at the nuclear membrane (Fig. 4d and Fig. 4e). We observed the least nuclear deformation in regions of highest lamin A expression and maximum deformation at regions of lowest lamin $\mathrm{A}$ expression. The ratio of applied osmotic stress to local nuclear deformation as a function of the lamin A expression yields the effective contribution of lamin A to nuclear stiffness; this reveals that the stiffness increases as lamin A expression increases locally (Fig. 4f). While this finding is consistent in all cells measured, some cells also presented low deformations in regions of low

206 lamin A expression (see supplementary information Fig. S6), which may be attributed to the 207 mechanical anisotropy of the underlying chromatin ${ }^{27}$. This may also contribute to the observed mechanical anisotropy of the nucleus along the major and minor axes of the nucleus, where the 

anticorrelation could be due to the effect on the underlying chromatin.

a

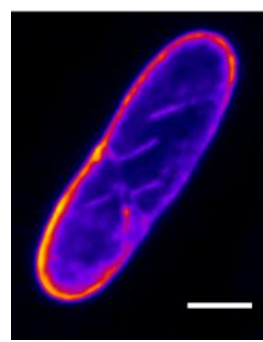

b

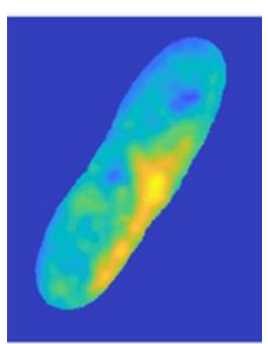

C
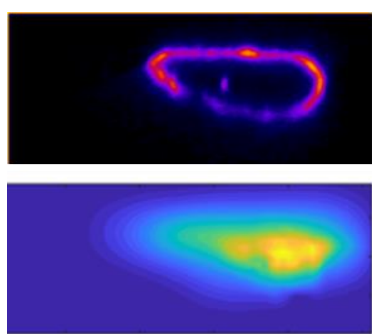

d

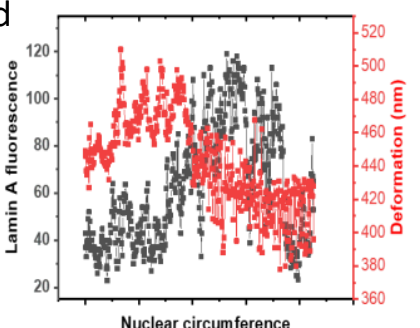

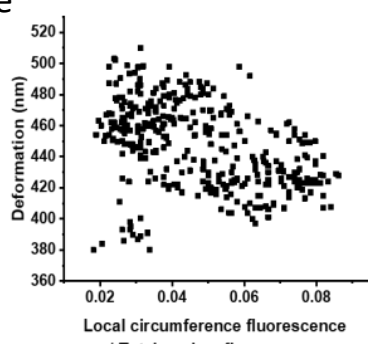

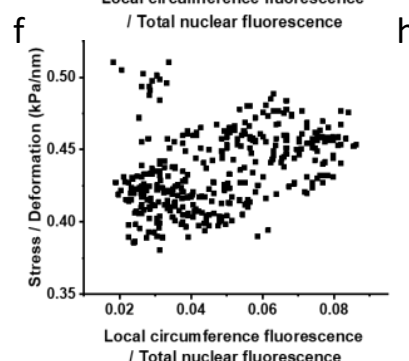

h g
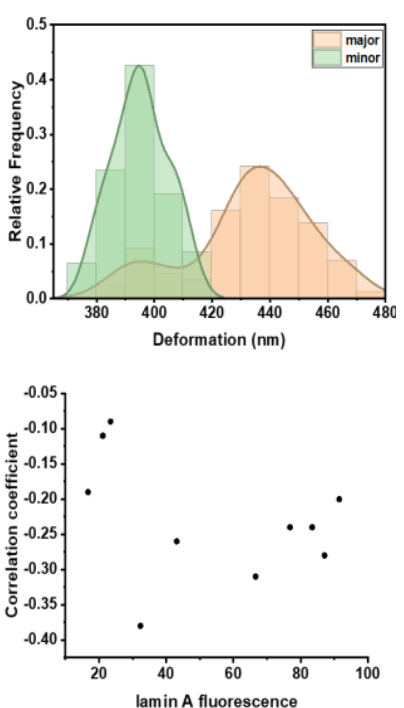

Figure 4 | Local nuclear deformation as a function of lamin A distribution in 3 T3 fibroblasts. a, Lamin A distribution along the nuclear membrane in X-Y plane. b, Example strain X-Y plane map of a 3T3 nucleus under 936.5kPa stress $($ scalebar $=5 \mu \mathrm{m})$. c, Lamin A distribution (top) and $\mathrm{Y}-\mathrm{Z}$ strain map (bottom) along the nuclear membrane. d, Lamin A fluorescence and nuclear deformation along the nuclear membrane. e, Deformation plotted against the ratio of local lamin A fluorescence at the membrane and total nuclear fluorescence. $\mathbf{f}$, Ratio of stress to deformation (effective stiffness) plotted against the ratio of local lamin A fluorescence at the membrane and total nuclear fluorescence. $\mathbf{g}$, Statistical distribution of overlaid major and minor axis. $\mathbf{h}$, The correlation coefficient between local deformation and local lamin A fluorescence was negative showing anticorrelation but with no relationship with total lamin A expression.

Discussion: Here, we have quantitatively examined the role of lamin A in nuclear mechanics. Using osmotic pressure, we applied precisely controlled stresses and measured global volumetric changes. We find that the overall bulk modulus of the nucleus increasing with expression levels 
227 of Lamin A. Decomposing nuclear compression, we identify anisotropic deformation of the 228 nucleus showing maximum deformation in z-axis followed by minor axis and then major axis, 229 respectively. Inspecting the distribution of lamin A more closely, we found distinct spatial 230 variations in concentration within the nuclear envelope; interestingly, the heterogeneity of lamin

231 A density increased with overall expression level. We suspected that these spatial variations in 232 concentration would lead to similar spatial variations in nuclear stiffness. By quantifying the 233 spatial variations of lamin A density and comparing this with a sub-micron resolution deformation 234 map of the nucleus, we indeed found a strong inverse correlation between local sub-micron lamin 235 A concentration and nuclear deformation, demonstrating that the spatial variation of lamin leads to heterogeneous nuclear membrane deformation. Also, the local deformations along the minor 237 axes of the nuclei were found to be larger than the corresponding major axes, which was consistent 238 with our previous observation, as seen in the bulk deformation of the nuclei.

239 The expression and distribution of lamin A are critical in nuclear mechanics, with variations or abnormalities in lamin A affecting nuclear stiffness leading to altered nuclear deformation.

241 Pathological conditions such as $\mathrm{HGPS}^{28}$ and $\mathrm{EDMD}^{29}$ are related to a mutation in the lmna gene 242 suggesting a potential role of nuclear deformation in nuclear mechanotransduction in the cell, but 243 the relationship between nuclear deformation and how it changes the gene expression is not clear. 244 The strongest evidence to this hypothesis comes from a recent study, where it is shown that when 245 the nucleus is physically compressed, there is translocation of an enzyme Histone deacetylase 3 246 (HDAC3) inside the nucleus. The role of this enzyme is to remove the acetyl group present on 247 histone residues which neutralize the positive charges present on the histones and inhibits its 248 interaction with the negatively charged DNA resulting in an open conformation of chromatin 249 known as euchromatin (non-compact expressing chromatin conformation). Thus, when the nucleus 
250 is mechanically compressed, HDAC3 translocates in the nucleus to remove the acetyl group on

251 the histone leading to a tighter histone-DNA interaction promoting the non-expressing

252 heterochromatin (highly compact non-expressing chromatin) conformation of the chromatin ${ }^{30}$.

253 Another factor contributing to nuclear stiffness is the tethering of chromatin itself with the nuclear

254 lamina $^{31}$. Since the chromatin can be present as heterochromatin and euchromatin, it contributes

255 to the mechanical anisotropy of the nuclear envelope ${ }^{32}$. While lamin A dominates nuclear stiffness

256 under high mechanical stress, under small stresses, chromatin has a crucial role as well ${ }^{27}$. More

257 condensed heterochromatin regions are stiffer and experience less deformation compared to

258 euchromatin ${ }^{33}$, making chromatin dynamics additionally important in nuclear

259 mechanotransduction. Nevertheless, the physical association between the nuclear lamina and the

260 chromatin, as well as the relationship between nuclear deformation and its direct effect on

261 epigenetic changes are still unclear and could prove to be essential in relating nuclear mechanics

262 with various pathological conditions.

263 Previous mechanotransduction studies have shown that mechanical forces can lead to translocation

264 of mechanosensitive proteins such as YAP from the cytoplasm to the nucleus under nuclear

265 tension $^{34}$, which affects properties like cell proliferation and differentiation in stem cells. Lamin

266 A could prove to be a critical factor in elucidating this relationship as it directly affects the nuclear

267 stiffness and its deformation. By manipulating lamin A expression, relating the change in nuclear

268 mechanics, and measuring downstream effects, we may better understand how nuclear stiffness

269 determines cellular fates, thus providing novel mechanics-centered strategies to correct defects in

270 diseases.

271 Acknowledgments: The authors thank members of Christian Frank's lab, University of

272 Wisconsin, for assistance with MATLAB FIDVC. AJE acknowledges support from NSERC 


\section{References}

276 1. Lamond, A. I. \& Earnshaw, W. C. Structure and function in the nucleus. Science 280, 547-553 (1998).

$277 \quad$ 2. Kirby, T. J. \& Lammerding, J. Emerging views of the nucleus as a cellular mechanosensor. Nat. Cell Biol.

278 20, 373-381 (2018).

279 3. Chen, J. et al. Cell mechanics, structure, and function are regulated by the stiffness of the three-dimensional 280 microenvironment. Biophys. J. 103, 1188-1197 (2012).

281 4. Vaziri, A. \& Mofrad, M. R. K. Mechanics and deformation of the nucleus in micropipette aspiration 282 experiment. J. Biomech. 40, 2053-2062 (2007).

5. Caille, N., Thoumine, O., Tardy, Y. \& Meister, J. J. Contribution of the nucleus to the mechanical properties

6. Adele Khavari, A. J. E. Nuclei deformation reveals pressure distributions in 3D cell clusters. PLoS One

7. Chu, F.-Y., Haley, S. C. \& Zidovska, A. On the origin of shape fluctuations of the cell nucleus. Proc. Natl.

8. Pajerowski, J. D., Dahl, K. N., Zhong, F. L., Sammak, P. J. \& Discher, D. E. Physical plasticity of the

9. Lin, H.-H. et al. Mechanical phenotype of cancer cells: cell softening and loss of stiffness sensing. Oncotarget 6, (2015).

294 11. Hetzer, M. W. The nuclear envelope. Cold Spring Harb. Perspect. Biol. 2, a000539 (2010).

12. Dechat, T., Adam, S. A., Taimen, P., Shimi, T. \& Goldman, R. D. Nuclear Lamins. 1-22 (2010).

13. Lammerding, J. et al. Lamin A / C deficiency causes Tema Grupo defective nuclear mechanics and mechanotransduction. J. Clin. Invest. 113, 370-378 (2004).

14. Hanson, L. et al. Vertical nanopillars for in situ probing of nuclear mechanics in adherent cells. Nat. Nanotechnol. 10, 554-562 (2015).

15. Guilluy, C. et al. Isolated nuclei adapt to force and reveal a mechanotransduction pathway in the nucleus. Nat.Cell Biol. 16, 376-381 (2014).

16. Harada, T. et al. Nuclear lamin stiffness is a barrier to 3D migration, but softness can limit survival. J. Cell Biol. 204, 669-682 (2014).

17. Wang, Y., Jiang, J., He, L., Gong, G. \& Wu, X. Effect of lamin-A expression on migration and nuclear

18. Moir, R. D. et al. Review: The dynamics of the nuclear lamins during the cell cycle - Relationship between

19. Schirmer, E. C. \& Gerace, L. The stability of the nuclear lamina polymer changes with the composition of lamin subtypes according to their individual binding strengths. J. Biol. Chem. 279, 42811-42817 (2004). 
21. Barateau, A. et al. A novel lamin a mutant responsible for congenital muscular dystrophy causes distinct abnormalities of the cell nucleus. PLoS One 12, 1-18 (2017).

22. Lammerding, J. et al. Lamins a and C but not lamin B1 regulate nuclear mechanics. J. Biol. Chem. 281, 25768-25780 (2006).

23. Mayer, C. R., Arsenovic, P. T., Bathula, K., Denis, K. B. \& Conway, D. E. Characterization of 3D Printed Stretching Devices for Imaging Force Transmission in Live-Cells. Cell. Mol. Bioeng. 12, 289-300 (2019).

24. Zhou, E. H. et al. Universal behavior of the osmotically compressed cell and its analogy to the colloidal glass transition. Proc. Natl. Acad. Sci. 106, 10632-10637 (2009).

25. Guo, M. et al. Cell volume change through water efflux impacts cell stiffness and stem cell fate. Proc. Natl. Acad. Sci. (2017). doi:10.1073/pnas.1705179114

26. Bar-Kochba, E., Toyjanova, J., Andrews, E., Kim, K. S. \& Franck, C. A Fast Iterative Digital Volume Correlation Algorithm for Large Deformations. Exp. Mech. 55, 261-274 (2015).

27. Stephens, A. D., Banigan, E. J., Adam, S. A., Goldman, R. D. \& Marko, J. F. Chromatin and lamin A determine two different mechanical response regimes of the cell nucleus. Mol. Biol. Cell 28, 1984-1996 (2017).

28. Goldman, R. D. et al. Accumulation of mutant lamin A progressive changes in nuclear architecture in Hutchinson-Gilford progeria syndrome. Proc. Natl. Acad. Sci. U. S. A. 101, 8963-8968 (2004).

29. Håkelien, A. M., Delbarre, E., Gaustad, K. G., Buendia, B. \& Collas, P. Expression of the myodystrophic R453W mutation of lamin A in C2C12 myoblasts causes promoter-specific and global epigenetic defects. Exp. Cell Res. 314, 1869-1880 (2008).

30. Damodaran, K. et al. Compressive force induces reversible chromatin condensation and cell geometrydependent transcriptional response. Mol. Biol. Cell 29, 3039-3051 (2018).

31. Schreiner, S. M., Koo, P. K., Zhao, Y., Mochrie, S. G. J. \& King, M. C. The tethering of chromatin to the nuclear envelope supports nuclear mechanics. Nat. Commun. 6, 1-13 (2015).

32. Berendes, H. D. \& Keyl, H. G. Distribution of DNA in heterochromatin and euchromatin of polytene nuclei of Drosophila hydei. Genetics 57, 1-13 (1967).

33. Hampoelz, B. \& Lecuit, T. Nuclear mechanics in differentiation and development. Curr. Opin. Cell Biol. 23, 668-675 (2011).

34. Driscoll, T. P., Cosgrove, B. D., Heo, S. J., Shurden, Z. E. \& Mauck, R. L. Cytoskeletal to Nuclear Strain Transfer Regulates YAP Signaling in Mesenchymal Stem Cells. Biophys. J. (2015). doi:10.1016/j.bpj.2015.05.010 